\title{
Reflexiones sobre el concepto de Ciudad Saludable a partir de tres ciudades del Noreste Brasileño
}

\section{Reflections on the concept of healthy cities from three cities in Northeast Brazil}

Article Info:

Article history: Received 2021-03-29 / Accepted 2021-03-29 / Available online 2021-03-30

doi: $10.18540 /$ jcecvl7iss 1pp12121-01-12e

Oriana Yenahi Andrade Serrano

ORCID: https://orcid.org/0000-0003-1210-5275

Universidad Federal de Alagoas, Programa de Postgrado en Arquitectura y Urbanismo, Brasil

E-mail: oriandrade59@gmail.com

Isabela de Araújo Oliveira

ORCID: https://orcid.org/0000-0002-7541-1221

Universidad Federal de Alagoas, Programa de Postgrado en Arquitectura y Urbanismo, Brasil

E-mail: isabelacivil4@gmail.com

Thiago Gilney Ferreira Silva

ORCID: https://orcid.org/0000-0002-3329-1030

Universidad Federal de Alagoas, Programa de Postgrado en Arquitectura y Urbanismo, Brasil

E-mail: thiagogilneyarq@gmail.com

Ricardo Victor Rodrigues Barbosa

ORCID: https://orcid.org/0000-0003-4971-6037

Universidad Federal de Alagoas, Programa de Postgrado en Arquitectura y Urbanismo, Brasil

E-mail: rvictor@fau.ufal.br

\section{Resumen}

El presente artículo reflexiona sobre el concepto de ciudades saludables frente al repertorio encontrado en el ambiente urbano del Noreste Brasileño. Con el fin de evaluar la conformidad del espacio urbano construido y lo esperado de una ciudad saludable, según lo postulado por la Organización Mundial de la Salud (OMS), se realizó un levantamiento fotográfico en los municipios de Petrolina (PE), Arapiraca (AL) y Maceió (AL), y, con apoyo en la literatura se realizó un análisis comparativo de las fotografías generadas. Se observó que el modelo de desarrollo actual de las ciudades estudiadas está compuesto por prácticas coherentes y otras disonantes a lo esperado para una ciudad saludable. Asimismo, fue posible notar que la discontinuidad que genera el encuentro entre los espacios saludables y los espacios hostiles origina estratificación en el espacio urbano y en la sociedad que los habita. Finalmente se percibió la tendencia, principalmente en edificaciones recientes a mostrar indiferencia hacia prácticas urbanamente saludables. Esta realidad evidencia la importancia de agilizar el enfoque en los paradigmas sociales y la planificación urbana sobre la consciencia ambiental.

Palabras clave: Ciudad Saludable. Adaptación climática. Ambientes hostiles.

\begin{abstract}
This article brings reflections on the concept of healthy cities in view of the repertoire found in the urban environment of northeastern Brazil. In order to assess the conformity of spaces with what is expected from a healthy city, according to guidelines of the World Health Organization (WHO), a photographic survey was conducted in the municipalities of Petrolina (PE), Arapiraca (AL) and Maceió (AL). With the help of literature, a comparative analysis of the photographs generated was performed. It was observed that the current development model of the cities studied is composed of coherent practices and also practices that are different from what was expected for the construction of a healthy city. Thus, it was noted that the mismatch that exists between healthy and hostile spaces generates discontinuity and stratification in the urban space and in the society that inhabits it. It was
\end{abstract}


also possible to perceive the tendency of the urban buildings to move away from urban healthy practices, a reality that highlights the importance of social paradigms and urban planning aimed at environmental awareness.

Keywords: Healthy City. Climate adaptation. Hostile environments.

\section{Introducción}

En la definición de la Organización Mundial de la Salud (OMS), una ciudad saludable es aquella que coloca en práctica de modo continuo la mejora de su ambiente físico y social, utilizando todos los recursos de su comunidad, OMS (1995). Por su parte, Mendes (1996, p.233, traducción de los autores), asocia el concepto de ciudad saludable al de ciudad sustentable, considerando la salud como un producto social, expresado en la calidad de vida de los habitantes, y resultante de las "relaciones entre los procesos biológicos, ecológicos, culturales y económicos-sociales que acontecen en determinada sociedad y que generan las condiciones de vida de la población". Entonces, puede afirmarse que, cuando las relaciones entre los procesos que acontecen en la sociedad contribuyen con la mejora de la calidad de vida colectiva de la población y apoyan las interacciones entre ellos, se habla de una ciudad saludable. Mientras que, cuando acontece lo opuesto, y esos procesos no ocurren en armonía, se habla entonces de una ciudad enferma u hostil.

Los factores que influyen en la calidad de vida y el alcance de una ciudad saludable son variados. De acuerdo con la OMS (1995), para que una ciudad se vuelva saludable, en ella debe ser proporcionado: 1) Un ambiente físico limpio y seguro; 2) Un ecosistema estable y sustentable; 3) Alto apoyo social, sin explotación; 4) Alto grado de participación social; 5) Satisfacción de las necesidades básicas; 6) Acceso a experiencias, recursos, contactos interacciones y comunicaciones; 7) Innovación y diversificación de la economía local; 8) Orgullo y respeto por la herencia biológica y cultural; 9) Servicios de salud accesibles para todos; 10) Alto nivel de salud.

La adaptación de la ciudad al clima local, tiene un papel importante en la conquista de alguno de esos postulados, principalmente en aquellos relacionados directamente con sustentabilidad, participación social, adaptación al ambiente y acceso a los servidos básicos. Diversos estudios sobre desarrollo urbano actual de las ciudades, muestran cómo algunas propuestas, comunes ya en nuestro imaginario colectivo, van en la praxis en contra de los principios expuestos sobre ciudades saludables. (Moreira Júnior, 2010; Serpa 2013; Suertegaray, Basso e Verdum, 2000; Leal, 2012: Luz, 2018; Costa et. al., 2019).

De forma cada vez más frecuente, se encuentran practicas constructivas prototípicas o de vanguardia que desde su concepción se apoyan en el uso de herramientas artificiales, como por ejemplo aires acondicionados, para la obtención de confort interno, superponiendo éste al confort externo, y a la práctica de premisas saludables como la adaptación climática, el trabajo con elementos naturales y la relación interior-exterior del espacio habitado. El uso intensivo en fachadas del vidrio u otros materiales inadecuados al clima local, así como la construcción de edificaciones desintegradas al espacio urbano, la consolidación de espacios áridos sin vegetación, la constante impermeabilización del suelo y la falta de mantenimiento de los espacios públicos existentes, son algunos ejemplos comunes de esas prácticas.

La percepción de esa contradicción llevó a los autores a cuestionarse sobre cuales factores de la práctica urbana contemporánea en estas ciudades se contraponen al alcance de una ciudad saludable y cuál es el impacto o producto social que esas prácticas generan en la salud. Con base en lo anteriormente expuesto, este artículo tiene como objetivo reflexionar sobre cómo el modelo de desarrollo actual en las ciudades brasileñas, converge o no con los postulados señalados por la OMS para la consolidación de una ciudad saludable. Para ello, se tomó como objeto de estudio el repertorio urbano actual de tres ciudades ubicadas en el noreste de Brasil, dos ciudades de porte medio y una capital: Petrolina-PE, Arapiraca-AL y Maceió-AL, respectivamente.

La ciudad de Petrolina está situada en la región de Pernambuco, con clima semiárido, caracterizado por tener un balance hídrico negativo, fuerte insolación anual, temperaturas 
generalmente altas y régimen de lluvia escaso e irregular (Brito, Moura e Gama; 2007). El municipio posee $4.561,874 \mathrm{~km}^{2}$ de área (2017), una población estimada de 354.317 habitantes, y está localizado a $-40,50^{\circ}$ de longitud y $-9,39738^{\circ}$ de latitud (IBGE, 2020).

Por su parte la ciudad de Arapiraca está situada en la región agreste de Alagoas. Es la segunda mayor del estado en términos poblacionales, después de la Capital Maceió. Cuenta con aproximadamente 230 mil habitantes, de acuerdo con lo estimado por el IBGE, 2018 (Arapiraca, 2020). De acuerdo con Xavier e Dornellas (2012), este municipio se localiza en un área de transición climática, entre los climas tropical húmedo y tropical semiárido, teniendo así por definición un clima tropical semi-húmedo.

Finalmente, el municipio de Maceió, capital del Estado de Alagoas, está ubicado en la costa norte del estado, y tiene un clima tropical lluvioso (cálido y húmedo). Según IBGE (2020), la ciudad tiene una población estimada de 1.025.360 habitantes y una superficie de $509.320 \mathrm{~m}^{2}$. Según Melo (2009), las características térmicas y dinámicas del lugar han sido influenciadas por el casco urbano, "debido a los cambios provocados por el exceso de impermeabilización del suelo, aumento de superficies edificadas y alteración del régimen del viento" (p.6, traducción de los autores).

\section{Marco referencial}

Las ciudades se están convirtiendo con el paso del tiempo en la forma de hábitat humano más común, según ONU HABITAT (2018) hoy, el 55\% de la población mundial vive en ciudades y se estima que esta proporción aumente $13 \%$ para 2050, haciendo que, el desarrollo sostenible dependa cada vez más de la gestión adecuada de este crecimiento urbano, especialmente en los países de ingresos medios y bajos que liderarán el proceso.

Una de las más notorias consecuencias de esta agrupación es la visibilidad e intensificación de las diferencias, convirtiendo al entorno urbano en un definitorio de la existencia o no, de interacción de estas diferencias en el espacio. La producción del espacio urbano actual ha estado marcada por la segregación espacial, según Moreira Júnior (2010) es la barrera (representada por los muros y casetas de vigilancia de condominios residenciales) la que se está imponiendo en la realidad contemporánea, delimitando espacios y negando la convivencia entre las diferencias. La barrera crea un entorno urbano ideal separado de la ciudad real, esta ruptura entre grupos sociales y territorios construye espacios diferenciados y sin continuidad con la estructura socioespacial que la rodea, lo que resulta en un tejido urbano formado por bruscas diferencias y regiones aisladas (Salgueiro, 1998). Serpa (2013) también sostiene que la implementación de límites y barreras en la ciudad reduce sustancialmente la interacción social y espacial de los pobladores de la ciudad, y a la par una pérdida de interés por el espacio urbano público y/o colectivo.

Un ejemplo de desinterés sobre el espacio público se hace visible en el estudio de Barros y Virgilio (2003), sobre la ciudad de Londrina-PR, en ella se observó que, aunque es bien conocido el aporte positivo que otorgan las plazas al medio urbano, estas áreas se encuentran en su mayoría depredadas, utilizadas como puntos de prostitución, narcotráfico y depósito de basura. Evidenciando, no sólo la falta de planificación, sino también de mantenimiento, fiscalización y consciencia tanto de la población como del sector gubernamental. Esta falta de mantenimiento de los espacios públicos trae a su vez otro agravante, la disminución del número de árboles en las plazas, lo que expone la importancia de una mayor reforestación de las diferentes áreas públicas

Cuando son comparados ambientes con y sin vegetación, es posible percibir como la falta del verde en las calles tiene un efecto negativo en el entorno urbano. En un estudio de Martini, Biondi y Batista (2013), se observó una diferencia de 2,7 ${ }^{\circ} \mathrm{C}$ entre las temperaturas máximas de dos tramos de calle, uno arbolado (temperaturas más bajas) y otro sin arboles (temperaturas más altas), en la ciudad de Curitiba. - PR. Este mismo estudio mostró que la calle con vegetación tenía un valor de humedad relativa mínima de $7.2 \%$ más alto que la calle sin vegetación. Además, se observó una menor amplitud térmica en la calle arbolada. La misma ciudad también fue estudiada por Leal (2012), el estudio reveló un comportamiento similar a las islas de calor retratadas en la literatura, 
donde los lugares con mayor intensidad de ocupación humana y menor vegetación presentaron mayores temperaturas. Estas investigaciones demuestran la importancia de contar con regiones arborizadas en las ciudades, con un mayor número de áreas permeables y verdes, que funcionen como "Islas de Frescura Urbana" (término defendido por el autor) y que contribuyan a amenizar el microclima urbano.

En la misma línea es importante señalar qué, la reducción de la vegetación conduce a su vez a una reducción de la cobertura vegetal del suelo. En la forma de urbanización actual, la ocupación de esta superficie conlleva en su mayor parte a la impermeabilización y a la pérdida de biodiversidad. En consecuencia, se genera la degradación del recurso suelo, el aumento de la infiltración del agua de lluvia, riesgos de erosión, compactación y deslizamientos de tierra, así como de inundación de arroyos y calles (Suertegaray, Basso e Verdum, 2000). Martins (2014), también argumenta que en las ciudades donde hay muchas edificaciones y pavimento asfáltico, las áreas impermeabilizadas obligan al agua que sería absorbida por el suelo a drenar por los conductos, lo que, sin el tratamiento adecuado puede provocar inundaciones.

El suelo es uno de los principales recursos naturales, sin embargo, ante la vorágine del desordenado crecimiento urbano y las actividades humanas, su proceso natural de formación ha sido significativamente alterado, perdiendo relevancia en su relación con el desarrollo vegetal y humano. Una ciudad bien arbolada con interacción de suelos permeables puede, por ejemplo, tener un mejor control sobre el movimiento del agua. Según Paula (2004), la vegetación es un medio natural que debe ser cultivado porque, las áreas con alta presencia de árboles tienen ambientes en mejores condiciones que las áreas con grandes niveles de construcción y poca cobertura vegetal.

Los materiales utilizados en la construcción de edificios urbanos son otro factor importante para la adaptación al clima local y en consecuencia para la producción de una ciudad saludable, Santos (2008), afirman que el confort térmico es una condición que determina la satisfacción del individuo con el medio habitado, mostrando gran influencia en la salud humana. Por ello las fachadas como pieles y elementos de intercambio térmico entre el espacio interior y exterior merecen un estudio detallado.

Es de interés resaltar el uso de materiales especulares o altamente reflexivos en las fachadas de los edificios, lo que conllevan a una mayor concentración de calor en la micro escala de la ciudad, elevando la temperatura del lugar tanto interna como externamente en relación con otras áreas urbanas. Adicionalmente, el uso de este tipo de fachada en zonas con alta incidencia solar puede ocasionar molestias visuales a los peatones en la calle, debido al deslumbramiento por el reflejo de los espejos, resultando en la ocurrencia de lugares con excesiva concentración de luz (Da Luz, 2018).

El crecimiento demográfico ha venido acompañado también de una creciente tendencia hacia propuestas arquitectónicas verticales y compactas, siendo en esta tipología, donde el uso de fachadas altamente reflexivas es más común. Esta realidad es una muestra de la clara necesidad de planificación que amerita este crecimiento.

Según Duarte (2012), además de establecer criterios de ocupación del suelo, el planeamiento urbanístico define las principales estrategias y políticas del municipio, especificando las restricciones y limitaciones que se deben aplicar, siempre con el objetivo de agregar comodidad y desarrollo a la población. En consecuencia, la falta de planificación de procesos urbanos de alto impacto, como la verticalización, por ejemplo, pueden derivar en ambientes confinados y desagradables, marcados por la acumulación de contaminantes que no son eliminados o suficientemente diluidos, a través del paso de luz y viento por aberturas.

Como indican Costa et al. (2019) las características constructivas del medio urbano influyen directamente en la calidad del ambiente interno y externo, y la contaminación, factor predominante en una ciudad insalubre, puede resolverse de manera colectiva con planificación, ya que su impacto refiere a toda la zona urbana y no solo una edificación (Santos et. al., 1992). 


\section{Metodología}

Para el desarrollo de este artículo se realizó un levantamiento fotográfico en las ciudades de Petrolina-PE, Arapiraca-AL y Maceió-AL con el fin de visualizar dentro de su repertorio urbano, los puntos coherentes y disonantes con la construcción de una ciudad saludable. Así, a partir de la revisión bibliográfica realizada previamente, la comprensión de los postulados que deben conformar una ciudad saludable y el registro de parte del repertorio urbano actual de las ciudades seleccionadas, se pretende reflejar qué características de estas ciudades son positivas y cuáles negativas desde el punto de vista de la salud ambiental urbana.

Asimismo, con el fin de facilitar el levantamiento fotográfico, y con base en la revisión bibliográfica realizada previamente, fueron establecidos los siguientes criterios de análisis:

- Adecuación de las edificaciones y el espacio urbano al clima local.

- Integración, continuidad y comunicación con el espacio urbano

- Áreas verdes y regiones de convivencia social;

- Permeabilidad del suelo

- Entorno físico limpio y seguro;

\section{Discusión y análisis de resultados}

El levantamiento fotográfico y la colección de imágenes creada permitieron la evaluación comparativa de la salud del ambiente urbano en las tres ciudades elegidas. Se identificaron repertorios constructivos coherentes e disonantes con el concepto de ciudad saludable, según lo postulado por la OMS. Se observó que, en general, estos modelos conviven, pero no siempre se comunican entre sí, lo que genera discontinuidades y estratificación palpable en la trama urbana. A los fines de este artículo, el repertorio fotográfico creado fue organizado en función de los criterios expuestos anteriormente.

\section{Adecuación de las edificaciones y el espacio urbano al clima local}

Las construcciones del Cuadro 1(A) muestran la existencia de edificios inadecuados para el clima cálido del noreste de Brasil, fue de interés que en las tres ciudades estudiadas se encontraron edificaciones con fachadas de cortina de vidrio, y con poco o ningún elemento protector de sombreado. Este tipo de envolvente arquitectónica es una característica común de los edificios contemporáneos, frecuentemente visible en sectores empresariales o turísticos de alta renta. Por su alta reflexión y conductividad térmica el uso intensivo del vidrio en climas cálidos, es una práctica que se aleja del concepto de salud en el entorno urbano. 


\section{Cuadro 1 - A) Edificaciones con elementos inadecuados al clima. B) Edificaciones con elementos adecuados al clima.}

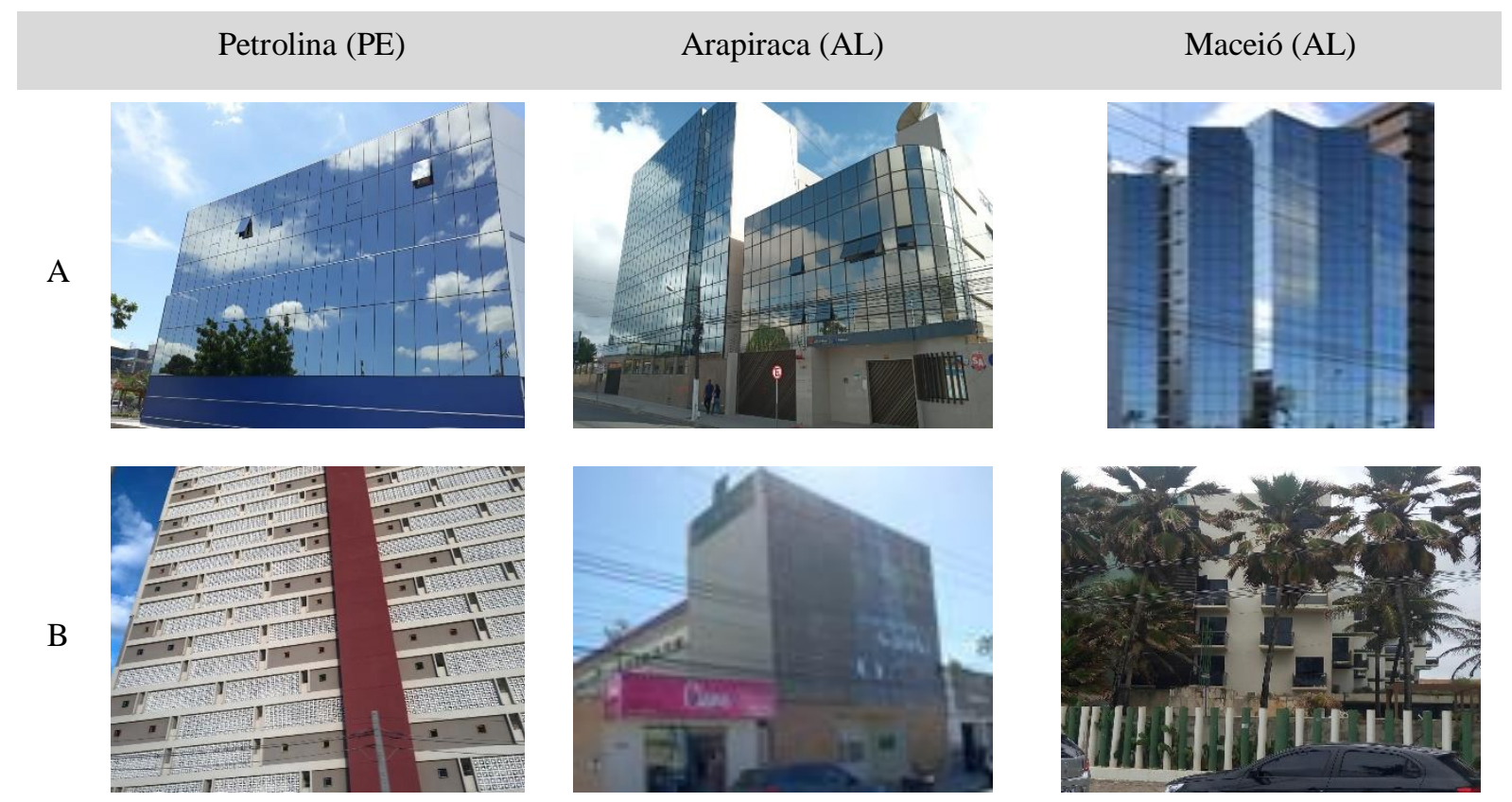

Fuente: Colección propia de los autores, 2020.

Aun así, en algunos casos puntuales, fue posible visualizar edificaciones con fachadas que interactúan de manera más armónica con el clima local, prácticas como la integración de muros calados (cobogós), balcones y vegetación como elementos de interacción con la luz solar y el viento, son ejemplos reflejados en el Cuadro 1(B). Sin embargo, cabe mencionar que, en muchos casos, estos elementos se asocian a espacios interiores con cerramientos de vidrio, por lo que se presume el confort térmico interno de estos edificios sea igualmente alcanzado en gran parte a través de medios artificiales. La idea de "confort ambiental" obtenido artificialmente es cuestionable, dado que, en estos casos para mantener la edificación adecuadamente ventilada e iluminada se provoca entre otras cosas, el calentamiento del ambiente externo a través de la salida de aire caliente de los acondicionadores, la reducción de la renovación del aire interno y el aumento en el consumo energético.

\section{Integración, continuidad y comunicación con el espacio urbano}

En cuanto a la integración, continuidad y comunicación con el espacio urbano, se observó una tendencia hacia el aislamiento de los espacios y la segregación social. Esto fue percibido a través de la constante presencia de grandes muros separadores, elementos de control y barrera principalmente en urbanizaciones, estas edificaciones comúnmente cerradas al exterior, están conceptualmente diseñadas para generar su propio confort y tener poca comunicación con el entorno, Cuadro 2(A). Estas prácticas constructivas perjudican sustancialmente la integridad urbana y la convivencia social, convirtiendo a la ciudad en un espacio hostil ajeno al concepto de ciudad saludable. 


\section{Cuadro 2 - A) Ausencia de continuidad en el espacio urbano. B) Continuidad en el espacio urbano.}

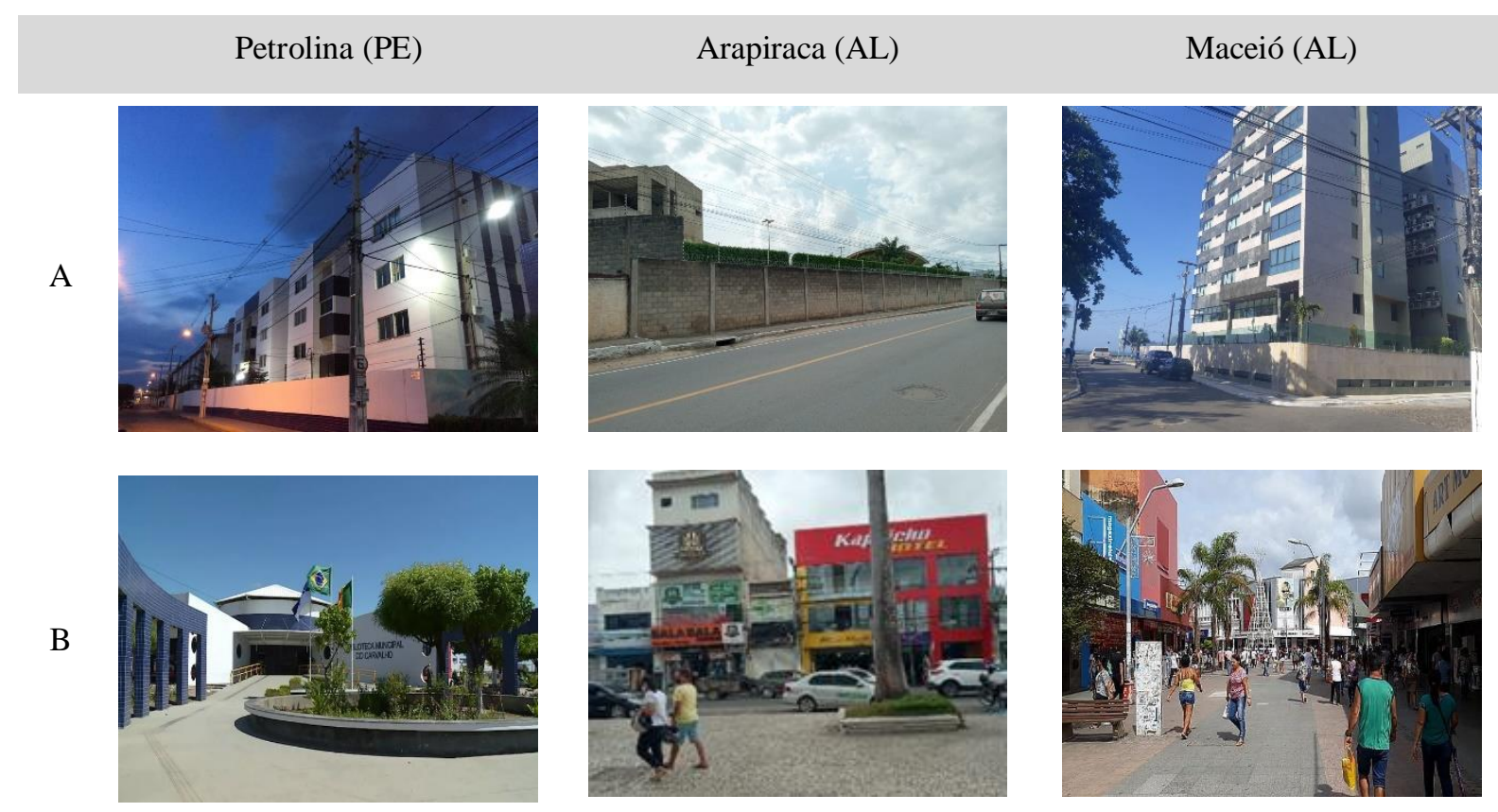

Fuente: G1, TNH1, Colección propia de los autores, 2020.

Fue posible notar también que, cuando existe alguna integración con el espacio urbano, es común que esta se consiga a través de espacios comerciales, por lo que cabe interpretar que, en el desarrollo urbano actual, el factor económico tiene mayor relevancia y potencial para la integración del espacio urbano que otros factores como la cultura, la seguridad, la diversificación o la participación social, los cuales son de gran importancia en una ciudad saludable.

Sin embargo, es importante resaltar que, en las ciudades analizadas, existen regiones del espacio urbano compuestas por prácticas saludables para la comunicación e integración social, como plazas y calles arboladas, visibles en el Cuadro 2(B). Se observó que estas prácticas, aunque existentes, no son suficientes ni constantes, convirtiéndose en puntos de "oasis" urbanos en medio de asfalto y concreto. La ciudad, analizada como un todo, no cuenta con la vegetación como elemento continuo en su formación.

\section{3. Áreas verdes y regiones de convivencia social}

Como se señaló en el tópico anterior, fue común encontrar en las tres ciudades poca arborización de los ambientes urbanos, Cuadro 3(A). Es pertinente acotar que ambientes pocos arborizados en climas cálidos, como los estudiados, llevan a condiciones climáticas urbanas y de confort inadecuadas que resultan en pérdida de calidad de vida de la población y disminución de la convivencia social.

Sin embargo, es importante resaltar también que en las tres ciudades fueron visibles plazas y regiones abiertas conservadas y en funcionamiento, Cuadro 3(B). Estas realidades, aunque puntuales, son representativas de la esperanza de fortalecimiento del entorno urbano, con espacios integrados de convivencia y continuidad social. Se evidenció en los tres casos estudiados que las regiones de mayor actividad social urbana, son a su vez las más arboladas y ambientalmente las más confortables, lo que en contraposición lleva a concluir que espacios hostiles, sin el confort de la vegetación, suelen ser espacios solitarios y por lo tanto inseguros, afectado directamente la convivencia y la salubridad de la ciudad. 


\section{Cuadro 3 - A) Regiones con poca vegetación y sin convivencia social. B) Regiones con vegetación y convivencia social.}

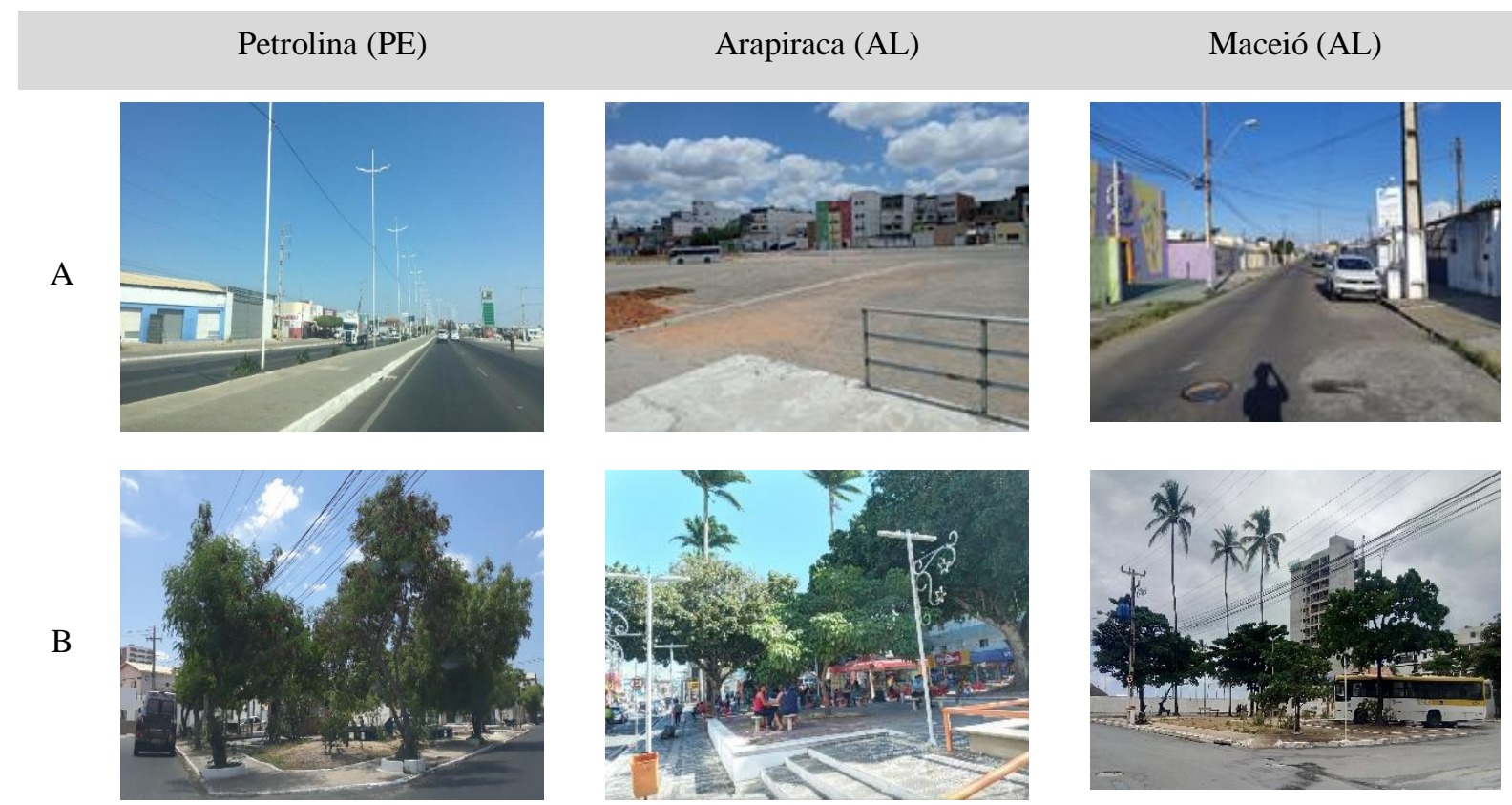

Fuente: Colección propia de los autores 2020.

\section{Permeabilidad del suelo}

El tipo de pavimento utilizado en los espacios urbanos es de gran importancia en cuanto a la capacidad del mismo para infiltrar el agua que en él se concentra, con el fin de reducir las posibilidades de inundaciones y, consecuentemente, los problemas económicos y sociales de la ciudad (Cooper, 2013). Desafortunadamente, en el modelo de desarrollo urbano actual los suelos de la ciudad son predominantemente impermeables.

Fue posible notar, Cuadro 4 (A), que en las tres ciudades son poco comunes calles con pavimento permeable, y que en la mayoría de las vías se utilizan materiales oscuros e impermeables, que contribuyen por una parte al aumento de temperatura en la ciudad, y por otra comprometen el drenaje, infiltración y humedad del suelo. 


\section{Cuadro 4 - A) Regiones con suelos impermeables y fallas de drenaje B) Regiones con integración de suelos permeables.}

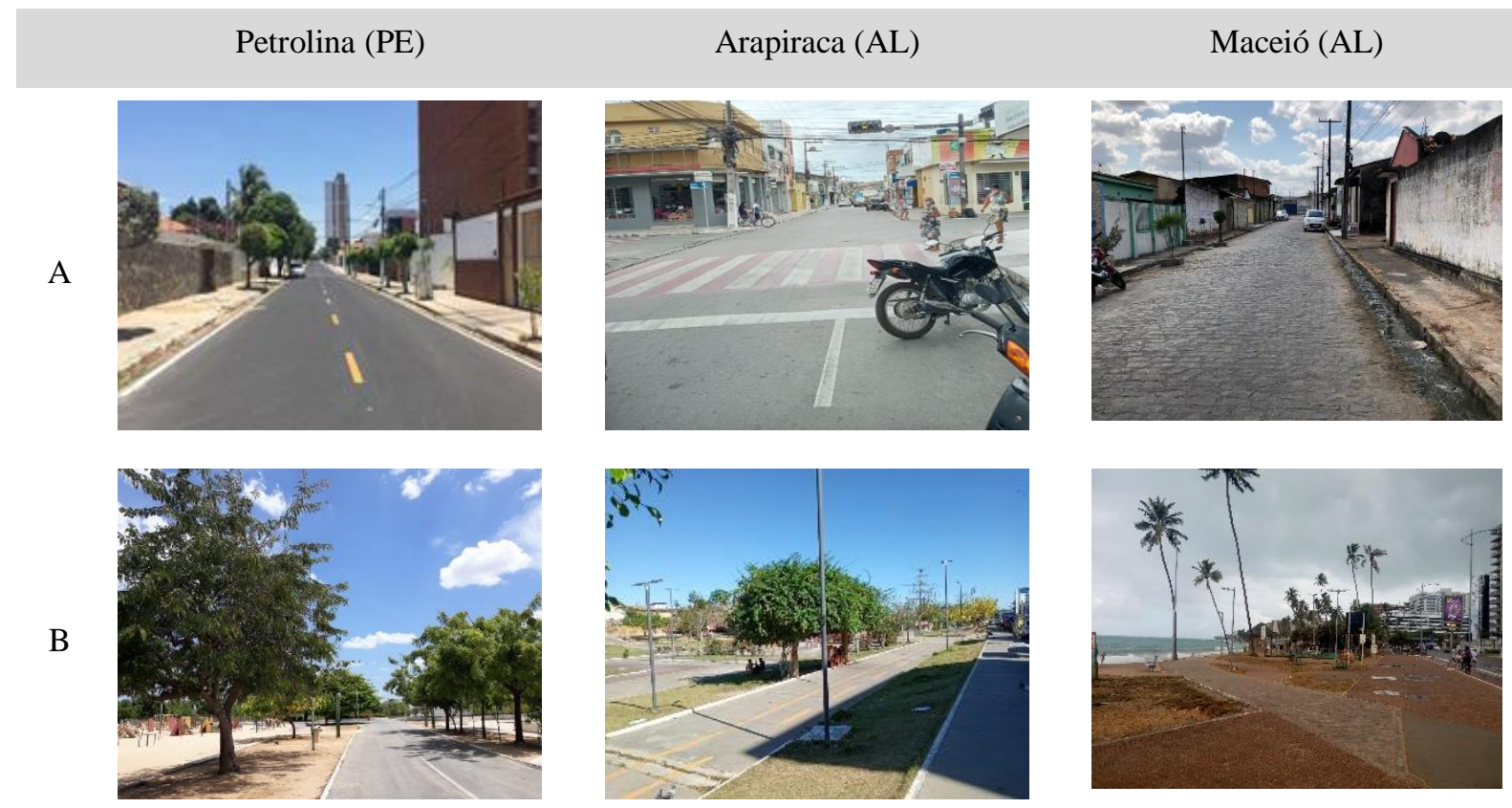

Fuente: Colección propia de los autores, 2020.

Zonas urbanas con suelos permeables, fueron encontradas en regiones específicas, mostrándose como la excepción a la regla en la ciudad, Cuadro 4 (B). Así, se observó que en su mayoría las áreas con suelos permeables se limitaban a plazas, islas centrales de las vías automovilísticas y a algunas calles de regiones periféricas, asociadas estas últimas a falta de inversión pública. Cabe destacar que, muchas de las vías públicas que contaban con una intervención constructiva reciente fueron pavimentadas con materiales impermeabilizantes. Hechos como este evidencian lo expuesto por Pinheiro y Souza (2017) sobre la necesidad de incorporar conceptos y prácticas de desarrollo sostenible en las áreas urbanas de hoy, donde el gobierno y la población tienen un rol fundamental.

\section{Ambiente físico limpio y seguro}

Otro aspecto de relevancia en la composición saludable de la ciudad es la existencia de entornos físicos limpios y seguros que permitan el uso continuo por parte de la población, y reduzcan la sensación de inseguridad. En las ciudades estudiadas se encontraron tanto ambientes acordes con este aspecto, espacios que estimulan el disfrute y contemplación, como se muestra en el Cuadro 5(A), como otros que lo contradicen, Cuadro 5(B). Se observó, con frecuencia en las áreas periféricas, grandes espacios baldíos cercanos a áreas habitadas y sin uso o propiedad aparente definida. La presencia continua de estos espacios, visualizados además en las tres ciudades, evidencian las fallas de planificación y participación social que envuelven el desarrollo urbano actual de las ciudades y generan estos grandes espacios sub-utilizados. 


\section{Cuadro 5 - A) Ambientes limpios y seguros. B) Ambientes abandonados e inseguros.}

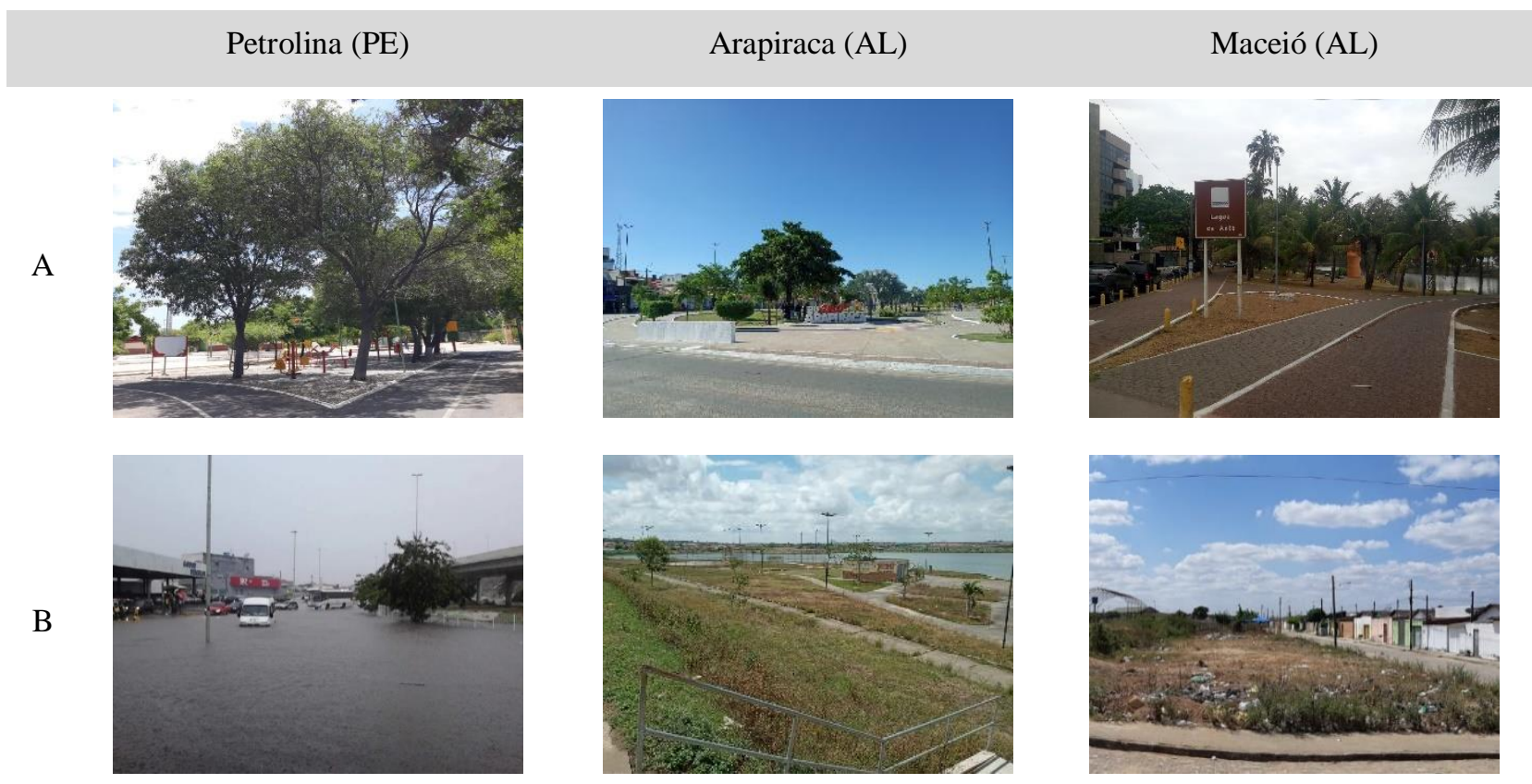

Fuente: G1, Colección propia de los autores, 2020.

De acuerdo con Souza y Rodrigues (2004), el urbanismo busca anticipar la evolución de los problemas y dificultades que se generan en la actualidad. La planificación no solo permite la macro visualización progresiva de la ciudad, sino que también conduce a la participación conjunta del Estado y la población civil, siendo este uno de los tópicos definidos por la OMS como importantes para el alcance de una ciudad saludable. Es evidente la necesidad de una planificación urbana que considere los aspectos ambientales y que incluya la vegetación en las áreas públicas de la ciudad, como parques, plazas y aceras (Amorim, 2011).

\section{Conclusión}

A través de la revisión de la literatura, la experimentación de la ciudad y el uso de fotografías como fuente de registro de información, se pudo notar que prácticas positivas y negativas de urbanización coexisten en el modelo constructivo actual de las ciudades estudiadas en el noreste de Brasil. Aun así, el elevado número de prácticas disonantes con el concepto de ciudades saludable evidencian el urgente y necesario cambio de paradigma constructivo y comportamiento por parte de la población, del poder público y del sector de la construcción, en escala urbana y arquitectónica.

Es de relevancia acotar que, aunque en la actualidad, es sabido que en climas cálidos el concepto de desarrollo saludable está contrapuesto al uso intensivo en fachadas de materiales altamente reflexivos como el vidrio, esta práctica continúa siendo común en el repertorio urbano de las tres ciudades. Asimismo, en cuanto a la participación de la vegetación en la composición urbana, aunque se ha demostrado ampliamente en numerosos estudios las connotaciones positivas que su uso genera en el espacio urbano, contradictoriamente esta práctica aún es menos común de lo que se necesita.

Lamentablemente, el registro realizado mostró que el repertorio construido actual, aunque en ocasiones consciente de su contexto, aún se encuentra alejado de los postulados de la OMS para las ciudades saludables. Es imperativo entonces, que se encuentren alternativas que permitan el crecimiento de las ciudades, sin comprometer el confort ambiental y la calidad de vida de los residentes.

Fue visible una creciente indiferencia por la adecuación climática principalmente en las edificaciones recientes, en las que aún se ejecutan practicas que privilegian la garantía del confort 
interno a través de medios artificiales, descuidan la relación con los elementos y espacios externos, potencian la construcción de espacios cerrados para facilitar el control, y favorecen el uso del espacio privado por encima del público. Esta realidad expone la necesidad de desarrollar acciones efectivas que regularicen y establezcan medidas de planificación urbanística que conlleven a mejores prácticas de vida e integración urbana, y proporcionen una mejor calidad de vida para los ciudadanos.

Se evidencia que en las ciudades estudiadas el encuentro y debate de dos modelos de desarrollo urbano es vigente: uno que viabiliza un entorno urbano saludable (asociado en ocasiones a una cultura antigua de interacción social) y otro, que privilegia la fragmentación del entorno urbano para el control del espacio. El estudio presentado permitió visibilizar también, cómo cada una de las decisiones expuestas sobre la ocupación del suelo están intrínsecamente relacionadas e interactúan favoreciendo o perjudicando la salud de la ciudad, lo que demuestra que, la elección del modelo que prevalecerá en las ciudades futuras es una decisión no solo de conocimiento, sino también de conciencia. Los dos modelos expuestos conviven en las ciudades y su desarrollo depende directamente del desempeño de la población, por tanto, es claro que la futura existencia de ciudades sanas, es al final una decisión.

\section{Agradecimientos}

Los autores desean agradecer a Capes y la OEA por su apoyo financiero; a La Universidad Federal de Alagoas y el Programa de Postgrado en Arquitectura y Urbanismo por el estímulo a la investigación.

\section{Referencias}

Adriano, J. R.; Werneck, G. A. F.; Santos, M. A. dos; Souza, R. de C. A. (2000). Construção de cidades saudáveis: uma estratégia viável para a melhoria da qualidade de vida? Ciência \& Saúde Coletiva, 5(1), 53-62. doi: http://dx.doi.org/10.1590/S1413-81232000000100006.

Almeida, J. R. de (2018). Planejamento urbano: uma abordagem sistêmica da interferência das áreas verdes na definição da qualidade de vida. Paisagem e Ambiente [S. l.], (41), 187-210. doi: https://doi.org/10.11606/issn.2359-5361.v0i41p187-210

Amorim, M. C. de C. T. (2011). Climatologia e gestão do espaço urbano. Mercator: Revista de Geografia da UFC, 9 (1), 71-90. doi: 10.4215/RM2010.0901.0005

Arapiraca. Prefeitura Municipal de Arapiraca. Dados Gerais. Arapiraca: 2020. Disponible en: <https://web.arapiraca.al.gov.br/a-cidade/dados-gerais/>. Consultado en: 12 de diciembre de 2020.

Barros, M. V. F.; Virgilio, H. (2003). Praças: espaços verdes na cidade de Londrina. Geografia (Londrina). 12(1), 533-544. doi: http://dx.doi.org/10.5433/2447-1747.2003v12n1p533

Brito; L. T. de L. Moura, M. S. B. de; Gama, G. F. B. (2007) Potencialidades da água de chuva no Semi-Árido brasileiro. Petrolina: Embrapa Semi-Árido.

Cooper, A. M. (2013). Estudo de viabilidade técnica da implantação de pavimentos permeáveis do tipo Infiltração Total para redução do escoamento superficial, na cidade de Alegrete/RS. Trabalho de Conclusão de Curso, Universidade Federal do Pampa, Alegrete, RS, Brasil.

Costa, R. F. W.; Rodrigues, M. A.; Rosa, T. C.; Silva, L. L.; Garcia, H. G.; Mello, J. S.; Souza, M. P. (2019). A qualidade do ar em ambientes comerciais fechados: prevenindo patologias associadas à permanência diária em espaços com climatização artificial. Revista Científica Doctum: Multidisciplinar, 1(2), 1-9.

Duarte, F. (2012). Planejamento Urbano. Curitiba: InterSaberes.

IBGE. Cidades. $\quad$ Petrolina: Disponible en: < https://cidades.ibge.gov.br/brasil/pe/petrolina/panorama>. Consultado en: 13 de diciembre, 2020. 
Leal, L. (2012). A influência da vegetação no clima urbano da cidade de Curitiba. Tese de Doutorado, Universidade Federal do Paraná, Curitiba, PR, Brasil.

Luz, I. B. da (2018). O impacto das ilhas de calor na escala gregária de Brasília com ênfase nas fachadas espelhadas. Relatório de pesquisa de Iniciação Científica, Faculdade de Tecnologia e Ciências Sociais Aplicadas, Brasília, DF, Brasil. doi: https://doi.org/10.5102/pic.n3.2017.5809

Maciel, C. D. R; Nogueira, M. C. J. A; Nogueira, J. D. S. (2011). Cobertura do solo e sua influência na temperatura de microclimas urbanos na cidade de Cuiabá - MT. Caminhos de Geografia, 12 (38), 40-57.

Martini, A.; Biondi, D.; Batista, A. C. (2013). Influência da arborização de ruas na atenuação dos extremos meteorológicos no microclima urbano. Enciclopédia Biosfera. 9(17), 1685-1695.

Martins, R. M. (2014). Análise da capacidade de infiltração do pavimento intertravado de concreto. Trabalho de Conclusão de Curso, Universidade Tecnológica do Paraná. Pato Branco, PR, Brasil.

Melo, J. D. de. (2009). Caracterização climática da cidade de Maceió como subsídio a decisões de planejamento. Dissertação de Mestrado, Universidade Federal de Alagoas, Maceió, AL, Brasil.

Mendes, E. V. (1996). Uma agenda para a saúde. São Paulo: Hucitec.

Moreira Júnior, O. (2010). Cidade partida: segregação induzida e auto-segregação urbana.Caminhos de Geografia. 13(33), 1-10.

OMS (1995). Veinte pasos para formular un proyecto de ciudades sanas; trad. HPP/HPL/95.3

Noticias ONU, Las ciudades seguirán creciendo, sobre todo en los países en desarrollo. New York, 2016. Disponible en: <https://www.un.org/development/desa/es/news/population/2018world-urbanization-prospects.html>. Consultado en: 13 de diciembre de 2020.

Paula, R. Z. R. (2004). A influência da vegetação no conforto térmico do ambiente construído. Dissertação de Mestrado, Universidade Estadual de Campinas, Campinas, SP, Brasil.

Pinheiro, C. R.; Souza, D. D. de. (2017). A importância da arborização nas cidades e sua influência no microclima. Gest. Sust. Ambient. 6(1), 67-82. doi: http://dx.doi.org/10.19177/rgsa.v6e1201767-82

Salgueiro, T. B. (1998). Cidade pós-moderna: espaço fragmentado. Território. (4), 39-53.

Santos, F. M. de M. (2008). Análise de desempenho térmico e lumínico em uma escola pública na cidade de Cuiabá/MT: estudo de caso. Dissertação de Mestrado. Universidade Federal de Mato Grosso, Cuiabá, MT, Brasil.

Santos, U. P.; Rumel, D.; Martarello, N. A.; Ferreira, C. S. W.; Matos, M. P. (1992). Síndrome dos edifícios doentes em bancários. Rev. Saúde Pública. 26(6), 400-404. doi: https://doi.org/10.1590/S0034-89101992000600005

Serpa, A. (2013) Microterritórios e segregação no espaço público da cidade contemporânea. Cidades. 10(17), 61-75.

Souza, M. L.; Rodrigues, G. B. (2004). Planejamento urbano e ativismos sociais. São Paulo: UNESP.

Suertegaray, D. M. A.; Basso, L. A.; Verdum, R. (2000). Ambiente e lugar no urbano: a grande Porto Alegre. UFRGS.

Xavier, R. A.; Dornellas, P. da C. (2012) Caracterização ambiental do município de Arapiraca, Região Agreste de Alagoas. Rev. Ambientale, 1(3), 108-121. 